\title{
Bases para escribir un trabajo cientifico
}

Por el doctor ALFONSO BONILLA-NAAR (")

\section{LISTA DE REVISTAS MMPORTANTES}

(Abreviatura y Dirección).

Según el Quaterly Cummulative Indox Modicus.

(Tomado de. Fishbein, Loc. Cit. Pág. 196-240).

So transcribe para aquéllos que no tionen ol Index Modicus a su alcance.

Acta cardiol. Acta cardiológica, Sociedad Bélgica de Cardiología. 80, rue Mercelis, Bruxelles.

Acta chir. belg. Acta chirurgica belgica. 64, rue de la Concorde, Bruxelles.

Acta chir, Scandinav. Acta chirurgica Scandinavica. P. A. Norstedt \& Sö. ner, Stockholm.

Acta med. Scandinav. Acta medica Scandinavica. Stockholm.

Acta obst. et gynec. Scandinav. Acta obstetricia et gynecologica Scandinavica. Prof E. Ahlström, Karolinska sjukhuset, Stockholm, 60.

Acta oto-rhino-laryng. belg. Acta oto thino-laryngologica beigiáa. DT. P. Hennebert, rue Mercelis, 86, Bruxelles.

(") El autor: Profesor Agregado de Clinica Quirúrgica de la Facultad Nacional de Medicina; Presidente de la Asociación Nacional de Gastroenterologia;- Presidente de la Sociedad Médico-Quirúrgica Lembana Barreneche.
Acta paediat. Acta pediatrica. Prof. I. Jundell. Ed. 23. Artillerigatan, Stockholm.

Acta paediat. belg. Acta pediatrica belgica. 64, rue de la Concorde, Bruxelles.

Acta physiol. Scandinav. Acta physio logica Scandinavica. P. A. Norstedt \& Söner, Tryckerigatan 2, Stockholm. 2.

Acta radiol. Acta radiologica. P. A. Norstedt \& Söner, Stockholm.

Actas soc. de cir. de Madrid. Actas de la Sociedad de Cirugía de Madrid; Avenida de José Antonio, 34, Madrid.

Actual med. peruana. Âctualidad mó dica peruana. Casilla de Correo, número 536, Lima.

Am. Heart I. American Heart Journal. C. V. Mosby Co., 3207, Washington Blvd., St. Louis 3.

Am. J. Anat. American Journal of Anatomy. Wistar Institute, 35th St. and Woodland Ave., Philadelphia 4. 
Am. J. Digest. Dis. The American Journal of Digestive Diseases. Sandfield Publishing Co., 435 Lincoln Bank To wer, Fort Wayne, Indiana.

Am. J. Hyg. American Joumal of Hy. giene. 615 N. Wolte St., Baltimore 5 . Am. I. M. Sc. American Journal of the Medical Sciences. Lea \& Febiger, 600 S. Washington Sq., Philadelphia 6 . Am. I. Nursing. American Journal of Nursing. 1790 Broadwary, New York. 19.

Am. J. Ophth. American Journal of Ophthalmology. Ophtaimic Publishing Co., 837 Corew Tower, Cincinnati.

Am. J. Path. American Journal of Pathology. Dr. C. V. Weller, East. University Ave., Ann Arbor, Michigan. Am. J. Pharm. The American Journal of Pharmacy and the Sciences Supporting Public Hoalth. Philadelphia College of Pharmacy and Science, 43d St., Kingsessing and Wooland Aves., Philadelphia, 4.

Am. J. Phys. Antropol. American Journal of Physical Anthropology. Wistar Institute, 36 th. St. and Woodland. Ave., Philadelphia.

Am. I. Physiol. American Journal of Phisiology. American Physiological Society, Managing Editor. Dr. D. R. Hooker, 19 W. Chase St., Baltimore 1.

Am. 1. Psychiat. The American Journal of Poychiatry. The American Psychiatric Association, 9 Rockefeller Plaza: New York, 20.

Am. J. Psychol. The American Journal of Psychology, Morrll Hall, Cornell University, Ithaca, N. Y.

Am. I. Psychotherapy. American lournal of Psychotherapy. 16 West 77th St., New York 24.
Am. J. Pub. Health. American Journal of Public Health and the Nation's Health. 1790 Broadway, New York. 19.

Am. J. Roentgenol. American Journal of Roentgenology and Radium Therapy, Charles C. Thomas, 301-327. E. Lawrence Ave:, Springtield. Ill. Am. J. Surg. American Journal of Sur. gery, 49 W. 45th St., New York 19. Am. J. Syph., Gonor \& Ven. Dis. Amo rican Journal of Syphilis, Gonorthea and Veneral Diseases. C. V. Mosby Co., 3207. Washington Blvd, St. Louis 3.

Am. J. Trop. Med. American Journal of Tropical Medicine. Williams \& Wilkins Co., Mt. Royal and Guilford. Aves. Baltimore 2.

Am. Rev. Soviet Med. American Review of Soviet Medicine. Sociedad Americano-Soviética de medicina, $\mathbf{5 8}$ Park Ave., New York 16.

An. argent. de oftal. Anales argentinos de Oftalmología. Organo oficial de la Cátedra de Ottalmología (Fac de medicina de Rositrio) y de la Sociedad de Oftalmología del litoral. Hospital Centenario, Sala, 10. Rosario.

An. brasil. de dermat. e sif. Anais brasileiros de dermatología e sifilografia. Caixa Postal 389, Río de Janel. ro. .

An. Brasil de ginec. Anais brasiletros do ginecologia. Cenxa Postal 1289. Río de Janeiro.

An. de cir. Anales de cirugra. Calle Paraguary 40, Rosario, Argentina.

An. Fac. med., Lima. Anales de la Facultad de medicina. Casialla 529 , Lima.

An. Fac. de med. de Montevideo. Ana les de la Facultad do Medictna do 
Montevideo. Facultad de medicina, Avenida General Flores, 2125. Montevideo.

An. Fac. de med. de Porto Alegre. Anais de Facultad de medicina de Porto Alegre. Caixa Postal número 657. Porto Alegre, Río Gronde do Sul, Brasil.

An. Fac. med. da Univ. de Sao Paulo. Anais de Facultad de Medicina da Universidade de Sao Paulo, Caixa Postal, 100-B, Sao Paulo.

An. med., México. Analocta médica. Organo oficial de la Sociedad médl. ca del Sanatorio español. Boulevard Miguel de Cervantes Saavedra. Mé xico, D. F.

An. paulist. de med. e cir. Anoris parulistas de medicina e cirugia. Caixa Postal, 1574, Sao Paulo.

An. Soc. biol. Bogotá. Anales de la Sociedad de Biología de Bogotá, ca lle 22, número 14.

An. Soc. méd. quir. d. Guayas. Anales de la Sociedad médico-quirúrgica del Guaryas. Apartado de Correo 1134, Guaryaquil, Ecuador.

An. Soc. mex. de oftal. y oto. rinolaring. Anales de la Sociedad mexicana de oftalmología y otorrinolaringología. Escuela nacional de medicina, esq. Brasil y Venezuela, MéxiCo, D F.

An. Soc. puericult. Buenos Aires. See Rev. Soc. puericult. Buenos Aires. Anesth. \& Analg. Current Regearches In Anesthesia and. Analgesta. 318 Hotel Westlake. Rockey. River 16. Ohlo.

Anesthesiology. Anesthesiology. The Journal of the American Society of Anesthesiologists, - Inc. Dr. William G. Schmidt, 235 N. 15 th, Philadelphila 2.
Ann. Allergy. Annals of Allergy including Spanish summaries); Official Journal of the American College of Allergists. 401. La Salle Medical Bldg. Minneapolis 2.

Ann. d'anat path. Annales d'amatomie pathologique et d'anatomie normale médico-chirurgicale. Masson \& Cie., 120 Boulevard Saint-Germain. Paris 6.

Ann. de dermat. et syph. Annales de dermatologie et de syphiligraphie. Bulletin de la Societé francorise de dermatologle et de syphilignaphie. Masson \& Cle., 120 Boulevard Saint: Germoni, Paris 6.

Ann. Inst. Pasteur. Annales de l'Institut Pasteur. Masson \& Cle, 120 Boulevard Saint-Germain. Paris 6.

Ann. Int. Med. Annals of Internal Mo dicine, Americon College of Phisicians, Prince and Lemon Sts. Jamcaster, Pa.

Ann. Ist. Carlo Forlanini. Annall dell' Istituto .Corlo Forlominis. Monte verde Nuevo. Roma.

Ann. med. Anales de médicine. Ma. sson \& Cle., 120 Boulevard Saint. Germain, Paris 6.

Ann. ostet. e ginec. Annall d'ostetri. cia e ginecologia. Via Commends, 12, Milomo.

Ann. d'oto-laryng. Les annales d'otolaryngologie. Bulletin de la Societé de laryngologie des hospitaux de Paris. Masson \& Clo, 120 Boulevard Satnt-Germoin, Paris 6.

Ann. de parisitol. Annales de parasltologle humatine ef comparíe. Mass son \& Cle., 120 Boulevard SointGermoin, Paris, 6.

Ann radiol diag. Annali di radiolo gla diagnostica. L. Cappolli, Via Fartni, 6, Bologna. 
Ann Kev. Elochem. Annual keview of Riochemistry. Annual Reviews Inc., Stanford University P. O., Calif. Ann. Rev. Physiol. Annual Review of Physiology. Annual Reviews, Inc., Stanford University P. O., Calif.

Ann. Rhoumat. Dis. Annals of the

Rhenumatic Diseases. H. K. Lewis \&

Co. Ltda., 136 Gower St., London, W. C. 1.

Ann. Surg. Annals of Surgery. J. B. Luppincott Co., 227-231 S. 6th St., Philadelphia 5.

Ann. Trop. Mod Annals of Tropical Modicine and Parasitology. University Press, 177 Brownlow Hill, Liverpool, 3.

Ap. respir. y tuberc. Santiago. Aparato respiratorio $y$ tuberculosis. Co rreo 11. Santiago de Chile.

Arch. antrolol. cim. Archivo di antro logía. criminale, psichiatria e mo dicina legale. Bocca, Via Durini, 31, Milano.

Arch. argent. de enferm. d. ap. digest. $y$ de la nutrición. Archlvos argentnos de enfermedades del aparato di: gestivo y de la nutrición. Junín 845. Buenos Atres.

Arch. argent. de pediat. Archivos ar. gentinos de Pediatría. Belgrano 2563. Buenos Aires.

Arch. argent. de tisiol. Archivos argentinos de tisiología. Hospital Tomú, Buenos Atres.

Arch. Bjochem. Archlves of Bioche mistry, Academic Press, Inc., 125 E. 23ird St., Now York 10.

Asch. biol. Paris. Árchives de biolo gie. Masson \& Cle., 120 Boulevard Saint-Germain, Parls, 60.

Arch. brasil, de med. Archivos brasiletros de medicina. Rue Evaristo da Velga, 16, Rilo de Jameiro. banos de cancerología, Orguno oficial del Instituto de Radlum, Hospital eNtra. Sra. de las Mercedes. La Habana. Calle 21, Vedado, Habana.

Arch. Dermat. \& Syph. Archives of Dermatology and Syphilogy. American Medical Association, $535 \mathrm{~N}$. Dearbom St., Chicago 10.

Arch. F. Dermat. U. Syph. Archiv fur Dermatologie and Suphilis, Springer Verlag. OHC, Nolkerbastel 5, Wlen I.

Arch espoñ. urol. Archivos españoles de uralogía. Atocha, 115, Madrid.

Arch. F. exper. Path. u Pharmakol. Neunyn-Schmiedebergs Archiv fur experimentelle Pathologie und Pharmakologie. pringer Verlag. Links. trasse 22/24. Berlin, W. 9.

Arch. farm. y bioquím. Tucumán. Archivos, de farmacia y bloguímica de Tucumán. Facultad de farmacia y blocpímica, Universidad, naclo nad de Tucumán, A yacucho 482, Re pública Argentina.

Arch. histol. norm. y pot. Archlvos de histología normal y patológica. Ins. ttución Cultural Española, Buenos Aires.

Airch. Hosp. Santo Tomás. Archivos del Hospital Santo Tomás. Hospltad Santo Tomás, Panamá, R. do P.

Arat Inst cordiol Mérleo, Archlvoe del Instituto de cardiología de Mt. xico. Calzada de la Piedad 300, Me xdco, D. F.

Arch. Inst. clr. prov. Buenos Atres. Ar. chivos del Instituto de cirugia do la provincla de Buenos Atres. Re pública Argentina. 
Arch. Int. Med. Archives of Internal Medicine. American Medical Association, 535 N. Dearborn St. Chicago 10.

Arch. internat. de neurol. Archives internationales de neurologie. J. Peyronnet \& Cie., 33, rue Vivienne, Paris, $2 \mathrm{e}$.

Arch. ital. chir. Archivio italiano de chirugia. L. Cappelli, Via Farini, 6. Bologna.

Arch. ital. mal appa diger. Archivio italiano delle malattiedell cappatato digerentes. L. Compelli. Via Farini, 6 , Bologna.

Arch. itäl. pediat. e puericolt. Archivio italiomo di pediatrie puericoltura. L. Cappelli, Via Farini, 6, Bologna.

Arch. ital. urol. Archivio italiano di urologia. L. Capnelli, Via Farini, 6 , Bologna.

Arch. F. klin. Chir. Archiv fur klinische Chirurgie. Springer Vorlag. Linkstrasse 22/24, Berlin, W. 9.

Arch. d. mal. de l'app. digestif. Archives des maladies de l'apparcil digestif et des maladies de la nutrition. Masson \& Cie, 120 Boulevard Sant-Germain. Paris 6.

Arch. d. mal. du coeur. Archives des maladies du coeur et des valsseaux. J. B. Bailliére et fils, 19, rue Hautefeuille, Paris $6 \mathrm{e}$.

Arch. d mal. profess. Archives des maladies professionnelles, de medecine du travall et de securité sociale. Masson \& Cle., 120, Boulevard Saint-Germain, Paris, 6.

Arch. med. int. Archivos de medictna interna, Apartado 2.053, Habana. Arch oftal. Buenos Aires. Archivos de oftalmologíá de Buenos Atres. Perú 795, Buenos Aires.
Arch. Neurol. \& Psychiat. Archives of Neurology and Psichiatry. American Medical Association. 535 N. Dearborn St., Chicago 10.

Arch. ortop. Archivio di ortopedia. L. Cappelli, Via Farini, 6e. Bologna. Arch. ostet. a ginec. Archivo di ostetri. cia e ginecologia. S. Andrea delle Dame, 19. Napoli.

Arch. Otolaryng. Archives of Otolaryngology. American Medical Associa tion, 535 M. Dearborn St., Chicago 10.

Arch. Path. Archives of Pathology. American Medical Association; $\mathbf{5 3 5}$ N. Dearborn St., Chicago 10.

Arch. de pediat. d. Uruguay. Archivos de pediatría del Uruguay, Av. Agraciada 1464. Montevideo.

Arch. peruanos pat. y clin. Archivos peruanos de patologia y rlínica. Hospital "2 de Mayo. Departamento de patología. Lima, Perú.

Arch radiol., Archivio di radiologia. Casella Postale 147, Napoli.

Arch. Soc. argent. de anat. y pat. Archivos de la Sociedad argentina de ancitomia normal y patología. Santa $\mathrm{Fe}$ i171. Buenos Aires.

Arch. Soc. de biol. de Montevideo. Archivos de la Sociedad de biología de Montevideo. Casilla Correo, 567. Montevideo.

Arch. Soc. cirujanos hosp. Archivos de la Sociedad de cirujanos de hospital. Casilia 2843. Santiago de Chille.

Arch. Surg. Archives of Surgery. Ame rican Medical Association, $535 \mathrm{~N}$. Dearborn St., Chicago 10.

Arch. venezol. puericult. y pediat. Ar. chivos venezolanos de puericulture y pediatría. Apartado 1428, Coracas Arq. de biol. Arquívos de biología Rua Sao Luiz, 161, Sao Paulo. 
Arq. brasil de cir. e ortop. Arquivos brasileiros de cirugía e ortopedia. Rua de Imperatriz, 83, Recife, Pernambuco. Brasil.

- Arq. brasil de oftal. Arquivos brasilei. ros de oftalmologia. Caixa Postal 4086. S. Poulo.

Arq. brasil. uro!. Arquivos braslleiros de urologia. Sob os auspicios da Cadeira de urologia da Facultad de medicine da Universidade de Sao Paulo e do Colegio brasileiro de urologistas. Caiza Posta., 297, Saro Poculo.

Arq. de cir. e exper. Arquivos de cirugia clínica e experimental. Dept. de técnica cirúrgica e cirugia experimental da Faculdade de medicina da Universiajade de Sao Paulo, Caixa Postal 4066. Sao Paulo.

Arq. Inst. biol. Saro Paulo. Arquivos do Instituto biologico, Carixa Postal 119-A. Sao Paulo.

Arq. neuro-psiquiat., Sao Poulo. Arquivos de neuro-psiquiatra. Caixa Postal 3461. Soro Parto.

Australian J. Exper. Biol. \& M. Sc. Austrilian Journal of Experimental Blolo gy and Medical Science. University of Adelaride, Adelaide.

Bact. Rev. Bacteriological Reviews. Williams \& Wilkins Co., Mt. Royal and Guilford Aves, Baltimore 2.

Biochem. J. Biochemical Journal. Cambrige University Press, Bentley House 200 Euston Road, Londion. N. W. 1. (Americam Agent University of Chi- cago Press, 58., and Ellis Ave., Chicago 37).

Biol. med. Paris. Biologie médicale. Revue des sciences blologiques considerees dans leurs rapports avec la medecine. 21, rue Jean Goujon, Paris, 80.
Blood. Blood. The Journal of Hemato logy. Grune \& Stratton, Inc. 381 4 th ave, New York 16.

Bol. Acad. nac. de med. de Buenos Ajres. Boletin de la Academia nacional de medicina de Buenos Aires. Las Heras 3092, Buenos Aires.

Bol. Asoc. med. de Puerto Rico. Boletin de la Asociación médica de Puerto Rico. Avenida Manuel Fernández Juncos, Parada 19, Santurce, San Juan.

Bol. Asoc. med. Santiago. Boletín de la Asociación médiza de Santiago. Calle José Trujillo Valdez No 133. Santiago de los Caballeros. Rep. Do minicana.

Bol. Clin. Univ. Antioquia. Boletín Clinico. Universidad de Antioquia. Organo de la Facultad de medicina de la Universidad de Antioquia. Medo llín, Colombia.

Bol. hosp. Caracas, Boletín de los hospitales civiles del distrito Federal. Apartado de Correos 1428, Caracas.

Bol. d. Hosp. policia nac. Boletin del Hospital de la pollicia naclonal. Oquendo y Estrella, Habana.

Bol. d. Inst. clín. quir. Boletín del Instituto de Clínica quirúrgica. Universidad de Buenos Aires. Paraguay 2150, Buenos Aires.

Bol. Inst. de med. exper. para el estud. -y trat. de cáncer. Boletín del Inetituto de medicina experimental para el estudio y tratamiento del cáncer With French, German and English summaries). Av. San Martín, 5481, Buenos Aires.

Bol. med.rocial. Santiago. Boletín mé dico-social de la Caja de seguro obligatorio. Casilla 7-D, Sontiago, Chille. 
Bol. Ofic. san. panam. Boletín de la Oficina sanitaria panamericama. (With occasional English translations). Washington, D. C.

Bol. San. Sao Lucas. Boletín do Sanatorio Sao Lucas. Suplemento de -Anais paulistas de medicina e cirugía. Rua Pirapitingui, 114, Sao Poulo.

Bol. Soc. chillena de obst. y ginec. Boletín de la Sociedad chilena de obs. tetricia y ginecología. Merced 565 , Santlago, Chille.

Bol. Soc. cir. Chile. Boletín de la Socie dead de cirugía de Chile. Merced 565. Santiago de Chile.

Bol. Soc. de cir. de Rosario. Boletines de la Sociedad de Cirugía del Ro sario. Librería y Editorial Ruiz, Córdoba 1.281, Rosario, República Argentina.

Bol. Soc. cir. d. Uruguary. Boletín de la Sociedad de cirugía del Uruguay. Agraciada 1464, Piso 13, Montevideo.

Bol. Soc. de obst. y ginec. de Buenos Aires. Boletín de la Socledad de obs tetricia y ginecología de Buenos Ai. res. Sonta Fe 1171, Buenos Aires.

Bol. y trab., Soc, argent. de.cirujamos. Boletines y trabajos, Sociedad argentina de cirujanos. Asociación médica argentina, Santa Fe 1171, Buenos Aires.

Brain. Brain A lournal of Neurology. Macmillan \& Company, Ltd. St. Mar. tin's St. London, W. C. 2. (Amerlcan office 60 Fifth Ave., New York 11). Brasil med.-Brasil-medico. Rua Rodrigo Sliva, 14 Río de Jomelro.

Brit. Heart. J. British Heart Journal. British Medical Association Torvistock Sq. London, W. C. 1.
Brit. J. Dermat. British Journal of Dermatology and Syphilis. H. K. Lewis \& Co., Ltd. 136 Cower St. London W: C. 1.

Brit. J. Exper. Path. The British Journal of Experimental Pathology. H. K. Le wis \& Co., Ltd. 136 Cower St., London, W. C. 1.

Brit. J. Ophth. The British Journal of Ophthalmology. Geo. E. Pulman \& Sons, Lid. 24 Thayer St., Marylebo ne, London W. 1.

Brit. J. Radiol. British Joumal of Radio logy. British Institute of Radiology. 32 Welbeck St., London, W. I.

Brit. J. Surg. British Journal of Surgery. John Wright \& Sons, Ltd., Bristol. (American agent - Williams \& Wilkins Co., Mt. Royal and Guilford Aves, Baltimore 2).

Brit. J. Tuberc. British Journal of Tuberculosis and Diseases of the Chest. Bailliére, Tindall \& Cox, 7 and 8 Henrietta St. London, W. C. 2.

Brit. J. Urol. British Journal of Urology. Count House, 28 Orchord St., Bristol 1.

Brit. J. Ven. Dis. The British Joumal of Veneral Diseases. Constable \& Co. 10 Orange St., Leicester Sq. London, W. C. 2.

Brit. M. Bull. British Medical Bulletin. Medical Departament. The British . Council, 3 Honover St., London, W. 1.

Brit. M. j. British Medicai Journal, 19 Tavistock St. London, W. C. 1.

Bull. Acad. Med. Toronto. Bulletin of the Academy of Medicine. 15 Queen's Park. Toronto.

Bull. Acad. nat. méd. Bulletin de l'Aca demie notionale de medecine Mas son \& cia. 120 Boulevard Saint-Ger main, Paris, 6. 
Bull. Acad. roy de méd. de Belgique. Bulletin de l'Academie royale de medecine de Belgique. Imprimerie Medicale ef Scientifique (Soc. An), 34, rue Botanique; Bruxelles.

Bull. Hist. med. Bulletin of the History of Medicine. Jonhns Hopkins Press. Balimore 18.

Bull. Inst. Pasteur. Bulletin de l'Institut Pasteur. Masson \& Cía. 120 Boulevard Saint-Germain, Paris $6 e$.

Bu!l. Johns Hopkins Hosp. Bulletin of the Johns Hopkins Hospital. Jonhs Hopkins Press, Baltimore 18.

Bull. New York Acad. Med. Bulletin of the New York Academy of Mo dicine, 2E. 103rd St., New York 29.

Bull. Soc. franc. d'hist. de la méd. Continued as Mem. Soc. franc. d'hist. med.

Bull. Tulane M. Fac. The Bulletin of the Tulane Medical Faculty $1430 \mathrm{Tu}$. lane Ave. New Orleans 13.

Bull. U. S. Army M. Dept. The Bulletin of the U. S. Army Medical Departament. Book Shop. Medical Fleld Service School, Carlisle Barracks, Pa.

Canad. J. Rosearch. Camadien Journal of Research. National Research Council of Canada, Otowa.

Cancer Research. Cancer Research. The Oflicial Organ of the American Association for Cancer Research. R. W. Briggs, Business Mgr., 1500 Greenmount Ave, Boitumore 2.

Chinese M. J. The Chinese Medical Joumal. 41 sekj Lu, Shanghal, China.

Cir. y cirujamos. Cirugía y Cirujanos. Academia Mexicana de Cirugia, Apartado 7994, México, D. F.

Crón. méd., Lima La Crónica Médica. Apartado 2563, Lima.
Collect. Papers Mayo Cin. \& Mayo Found. Collected Papers of the Mayo Clinic and the Mayo Foundation. W. B. Scaunders Company, W. Washington sq. Philadelphia 5.

Día méd. El Dia Médico. Córdoba 2093, Buenos Aires.

Dis of Chest. Disenses of the Chest (incluiding Spanish summaries). 500 N. Dearborn St. Chicago 10.

Endocrinology. Endocrinology; Bulletin of the Association for the Study of Internal Secretions Charles C. Tha mas, 301327 E Lawrence Ave. Springtield, III.

Foord Reserarch. Food Reseanch ta rrard Press, Champarign. 111.

Gac. méd. de Caracas. Gaceta médica de Caracas. Organos de la Acadomia Nactonal de Medicina, Apartado de Correos, 804, Caracas.

Gac. med. españ. Gacota médica es pañola. Apartado 8026, Madrid.

Gac. med. de México. Gaceta médica de México. Apartado postal 8075 Méxdco, D. F.

Gastroenterology. Gastroenterology. Otticlal journal of the American Gastroenterological Association. Will. miams \& Wilkins Co., Mt. Royal and Guilford Aves. Boltimore 2.

Geriatrics. Geriatrics. Otticial joumal of the American Geriatrics Society, 84 South 10th. St., Minneapolis 2.

Glor. Hal anest. o ancig. Giomaie tla licmo di anesteda e dianalouda, Ca sella Poot. 491, Via Martiri dilla L. bertá, 15, Tortno.

Qy's Hosp. Resp. Cuy's Hooptal Re ports. Guy's Hospital, London Brddge, London, S. E. 1.

Hoja tritol. Hota tratolótica. Casilla do Correo, 2605, Montavideo. 
Hospital, London. The Hospital Official Organ of the British-Hospitals Asso ciation and of the Incorporated Asso ciation of Hospital Officers. Secretary, I. A. H. O., 12 Crosvenor Grescent. London, S. W. 1.

Hospitals. Hospitals. The Journal of the American Hospital Association, 18 E. División St. Chicago 10.

-Indian J. Surg: Indian Journal of Surgery. Soc'y. Association of Surgeons of India, Binfields, Kilpout, Madras.

Indian M. J. The Indian Medical fournal. Dr. Khan Chand Wohra; Treas., Chicharwatni, Dt. Montgomery, Punjab.

Indust. Med. Industrial Medicine (incluiding) Industrial Hygiene Section. 605 N. Michigan Ave., Chicago.

Inform. med. Habana. Informaciones médicas. Reina 310, Habana.

Internat. J. Leprosy. International Journal of Leprosy. Temporary Office: Dept. of Hygiene, School of Mediclne, Western Reserve University, Cle veland 6 Ohio.

J. A. M. A. Journal of the American Medical Association. 535 N. Dearborn St., Chicago 10.

J. Allergy. The Journal of Alergy. C. V. Mosby Co., 3207 Washington Blyd., St. Louis 3.

J. Anat. Journal of Anatomy. Cambrige University Press, Bentley House, 20 Euston Rd. London N. W. 1. (Americom agent University of Chicago Press, 58th St. and Ellis Ave., Chicago).

J. Aviation Med. The Journal of Avia. tion Medicine. The Bruce Publishing Co. 2642 University Ave., St. Poul.
J. Bact. Journal of Bacteriology. Williams \& Co., Mt. Royal and Guilford Aves., Baltimore 2.

J: Biol. Chem. Journal of Biological Chemistry. Americon Society of Biolo gical Chemist, Inc. Williams \& Wilkins Co., Mt., Royal and Guilford Aves., Baltimore 2.

J. Bone \& Joint Surg. The lournal of Bone and Joint Surgery. The Otficial Publication of the American Ortho. paedic Association. The British Orthopardic Association. The American Academy of Orthopaedic Surgeons. The Australian Orthopaedic Association, The Canadian Ortho paedic Association. 8 The Fenway, Boston 15; 45 Lincoln's Inn Fields, London, W. C. 2.

J. de chir. Journal de chirurgie. Masson \& Cie., 120 Boulevard Saint Germain, Paris, 6.

J. Clin Endocrinol. The Journal of Clinical Endocrinology. Charles C. Thomas, 301-327 E. Lawrence Ave., Springfield, 111.

J. Endicronol. The Journal of Endocrinology. Cambrige University Press.

I. Exper. Biol. The Joumal of Experimental Biology. Cambrige University Press, Bentley House, 200 Euston Rd. London N. W. L. (American agent-University of Chicago Press, 58th St. and Ellis Ave., Chicago).

J. Exper. Med. Joumal of Experimental Medicine, Rockfeller Institute for Médical Resecurch. York Ave, and 66th St., New York 21.

J. Genetics; Journal of Genetics. Cam. brige University Press, Bentley House, 200 Euston Ed. London N. W. 1. (American Agent-University of Chi. cago Press, 58th St., and Ellis Ave. Chicago). 
J. Frane. méd. et thorac. Journal trancaris de médicine et chirurgie thoracique. Gaston Doin \& Cie, 8 place de l'Odeon. Paris, $6 e$.

J. Helminthol. Journal of Herminthol. logy. The Institute of Agricultural Parasitology. Wiches Farm Drive, Hertield Road, St., Albans, England. Hist. Med. \& Allied Sc. Journal of the History of Medicine and Allied Sciences. Henry Schuman, $20 \mathrm{E}$. 70th St. New York 21.

1. Inmunal. Journal of Inmunology. Virus Research and Experimental Chemotherapy. Williams \& Wilkins Co., Mt. Royal and Guilford Aves., Bajtimore 2.

J. Indust. Hyg. \& Toxicol. Journal of Industrial Hygiene and Tecicology with Abstract of Literature. Williams \& Wilkins Co., Mt. Royal Guilford Aves., Baltimore 2.

J. Infec. Dis. Journal of Infectiou= Di. senses. University of Chicago Press, 5750 Ellis Ave., Chicago.

1. Internat. Coll. Surgeons. The Journal of the International College of Sur. geons. Dr. Max Thorek, Editor, 850 W. Irving Parck, Chicago.

3. Invest. Dermat. The Journal of Inves. tigative Dermatology Williams \& Wilkins Co., Mt. Royal and Guilford Aves., Baltimore 2.

1. Lab \& Clin Med. Journal of Labo ratory and Clinical Medcine C. V. Mosby Co., 3207 Washington Blvd. St. Louis 3.

I. de med. de Paris. Journal de medecine de Paris. 53 rue de la Proces. zion, $15 e$.

J. Mt. Sinar Hosp. Joumal of the Mount Sinar Hospital. I E. 100 th. St., New York.
J. Nat. Malaria Soc. The lournal of the National Malaria Society. P. O. Box 997. Tallahassee, Florida.

J. Neurosurg. Journal of Nenurosurgery. Charles C. Thomas, 301-327 E. Law. rence Ave., Springtield, 111.

J. Nutrition. The Journal of Nutrition. Wistar Institute of Anatomy and Biology, 36th St. and Woodland Ave., Philadelphia 4.

J. Palestine Arab M. A. The fournal of the Palestine Arab Medical Association. Mamilla Road Ne 36, Jerusalem, Palestine.

J. Parasitol. Joumal of Parasitology. New York University, Universtty Height ; New York 53.

J. Pediat. The Journal of Pediatrics. C. V. Mosby Co. 3207 Washington Blvd., St., Louis 3.

J. Pharmacol. \& Exper. Therap. Journal of Pharmacology and Experi. mental Therapeutics. Williams \& Wilkins Co., Mt. Royal and Gullort Aves., Baltimore 2.

I. de radiol el d'electrol. Joumal de radiologite et d'elantrologio. Massión \& Cle, 120 Boulevard Sarint-Germain. Paris, 6.

J. Thoracic Surg. The Journal of Thoracic Surgery C. V. Mosby Company, 3207 Washington Blvd., St. Louis 3.

J. Urol. The Journal of Urology. Wi. lliams \& Wilkins C., Mt., Royal and Guilford Aves. Boltimore 2.

Lahey Clin. Bull. The Lahey Clinic Bullettn. 605 Commonwealth Ave, Boston 15.

Lancet. Lancet. 7 Adam St. Adelphi, London W. C. 2; Oxford University Press (Amertican Branch), 114 Fifth. Ave, Now York 11. 
Laryngoscope. Laryngoscope. 640 S. Kingshighwory. St. Louis 10.

Leposy. Rev. Leposy Review. The Bristish Empire Leprosy Relief Association 25 Kidderpore Ave., London, N. W. 3.

Lyon Chir. Lyon Chirurgical. Masson \& Cie., 120 Boulevard Saint-Germain. Paris 6 .

Lyon med. Lyon médical. 12 rue de la Borre, Lyon.

M. Clin. North America. The Medical Clinics of North Americana, W. B. Scounders Co., W. Washington Sq. Philadelphia 5.

Med. y cir., Bogotá. Revista Medicina y Cirugía de la Lombana. apt, 276. Bogotá.

Med. clin. Barcelona. Medicina clínica. Organo de la Facultad de medicina, hospitales y sociedades médicas de Barcelona. E. Granados, 121, Barcelona.

Med. espñ. Medicina española G. V. Fernando el Católico, 27, Pral. Valencla.

Medicina, México. Medicina. Revista mexicana, Av. Yucatán 29, México, D. F.

Mem. Inst. Butantan. Memorias de Instituto Butantan (With English abstractos). Caixa Postal 65, Sao Paulo.

Mem. Inst. Oswaldo Cruz. Nemorias dó Instututito Oswaldo Cruz. Caixa postal 926, Río de Janeiro.

Monde med. Paris. Le monde médical. Revista mtemacional de medicina $y$

- terapéutica (Sapanish edition) Calle Balmes 209-211, Barcelona; Casilla 8. Sucursal 5 (Caballito), Buenos Atres.

Mod. Hosp. Modern Hospital. 919 N. Michigan Ave. Chicago 11.
Mem. Soc. franc. d'hist. med. Memo ries de la Societé francaise d'histoire de la médecine et de ses filiales. 66. Boulevard Raspail, Paris $6 e$.

Nat. Inst. Health Bull. National Institute of Health Bulletin. Federal Security Agency U. S. Public Health Service, Washington, D. C. (Listed under Books).

New England J. Med. New England Journal of Medicine. Published by the Massachusetts Medical Society. 8 The Fenwary. Boston 15.

New Orleans M. \& S. J. New Orleans Medical and Surgical Joumal. 1430 Tulane Ave., New Orleans 13.

New York State J. Med. New York State Journal of Medicine, 292 Madison Ave., New York 17.

Obst. y ginec. latino-am. Obstetricia y ginecologia latinoamericanas. Calle José E. Uriburu 1578, Buenos Aires. Occup. Therapy. Occupational Therapy and Rehabilitation. Williams \& Wilkins Co., Mt.-Royal and Guilford Aves. Baltimore 2.

Ohio State M. J. Ohlo State Medical Journal. 79. E. State St., Columbus 15.

Otarhino-laryng. internat. L'oto-rhino laryngologie internationaie Librairi Arnette, 2 rue Casimir-Delavigne Paris.

Oto-rino-laring. ital. L'oto-rino-laringole gia italiana. L. Cappelli, Via Far ni, 6, Bologna.

Parasttology. Parasitology. Cambric University Press, Bentley House, 21 Euston Rd. London, N. W. I. (Am rlcan agent-Untversity of Chlcar Press, 58th St. and Ellis Ave, C cago).

Penneylvania M. I. Pensylvania Me cel Journal, 230 State St. Harriabur 
Pediat. Américas. Pediatria de las Américas. Ponciano Arriaga 6-2, México, D. F.

Philippine J. Surg. Philippine Journal of Surgery. Philippine General Hospi1al. Manila. Philippines.

Physiotherapy Rev. The Physiotherapy Review. American Physiotherapy Association 720 N. Michigan Ave., Chicago 11.

Plas! \& Reconstruct. Surg. Plastic and Reconstructive Surgery. The Wi. Illams \& Wilkins Co., Mount Royal \& Guillord Aves. Boltimore 2, Md.

Portugal med. Portugal medico. Rua Candido dos Reis, 47, Porto, Portugal.

Postgrad. Med. Post. graduate Medicine. Official Journal of the Interstate Postgraduate Medical Association. 512 Essex Eldg. Minnecpolis 2.

Prensa med. argent. La prensa médica argentina, Junín 845, Buenos Aires.

Presse med. La presse médicale. Masson \& Cie. 120 Boulevard Saint-Germain, Paris, $6 e$.

Proc. Roy. Soc. Med. Procedings of the

Royal Soctety of Medictne. Long. mans, Green \& Co., Ltd. of Paternostor Row, 43 Albert Drive. London, S. W. 19.

Proc. Nat. Acad. Sc. Procedings of the National Academy of Sclenes Constitution Ave. and 21 st St. Washing. ton 25, D. C.

Proc. Stal! Meet. Mayo Clin. Procee dings of the Staff Meetings of the Mayo Clinic. Roehenter, Minn.

Psychiatry. Psychiatri. Journal of the Biology and the Pathology of Inter. personal Telations. William Alanson White Psychatric Foundation, 1711 Rhode lsland Ave., N. W. Washing. ton 6. D. C.
Psychosom. Med. Psychosomatic Medictne. The American Soclety for Resecurch in Peychosomotic Pro blems, Inc., Madison Ave., New York, 21.

Puerto Rico J. Pub. Health \& Trop. Med. Puerto Rico Journal of Public Health and Tropical Medicine. (With Spanich translations). Columbia University Press, 2960 Broadwary, New York 27.

Radiologia. Radiología. Alstna 3117. Buenos Atres.

Radiology. Radiolooy. A. Monthly Journal Devoted to Alinical Radiological Radiology and Allied Salences $R$ a diological Society of North America. 607 Medical Arts Bldg. Syracuse 2. Rev. argent. de cardiol. Revista argentina de cardiología, Larrea 1132 , Buenos Aires.

Rev. argent. dermatosif. Revista argentina de dermatosifilología. EI Ato neo, Córdoba 2099, Buenos Alres

Rev. argent. de urol. Revista argentna de urologia, Charcas 1358, Bue nos Atres.

Rev. Asoc. argent. dietol. Revista de la Asociación argentina de dietolo gía. Calle Pueyrredón 2423, Buenos Aires.

Rev. Asoc. med. argent. Revisto de la Asociación médica argentina. Calle Santa Fe 1171, Buenos Atres.

Rev. brasill de biol. Revista brasileira de biología. Organo oticial da So cledado do bioloria do Bravil. Cat. xa postal 1.587. Río de Jamoiro.

Rev. chllena de hig. $y$ med. prev. Re viata chilena de higlene y medictna preventiva. Dirección General do Sanidad, Canllla 41D, Santlaso do Chillo. 
Rev. brasil. de leprol. Revista brasilera de leprología. 2a Serie da Revista de leprología de Sao Paulo Av. Dr. Arnaldo, 925, Sao Paulo.

Rev. clin. españ. Revista clínica espanola. Antonio Maura 13. Madrid.

Rev. cubana de cardiol. Revista cuba na de cardiologia. Apartado 2108. Habana.

Rev. españ. cir. Revista española de cirugia. Alocha, 115, Madrid.

Rev. españ. cir. tranimatol. ort. Revista española de cirugía, traumatología y ortopedia. Velásquez, $106 \mathrm{prl}$. Madrid.

Rev. españ. enferm. ap. digest. y nutrición. Revista española de las enfermedades del aparato digestivo $y$ de la nutrición F. G. Sicilia, Flora, 6. Madrid.

Rev. Fac. de med. Bogotá. Revista de la Facultad de Medicina. Calle 10 , número 13-99. Bogotá, Colombia.

Rev. Gastroenterol. The Revieq of Gastroenterology. Official Publication of the National Gastroenterological Association. 1819 Broadwory, New York 23.

Rev. gastro-enterol. de México. Revista de gastro-enterología de México. Plaza de la República, No 46, D. F.

Rev. Hosp. clin. Revista de Hospital das clínicas. Hospital dar Clínicas, Sao Paulo.

Rev. d. Inst. Salub. y enferm. trop. Re vista del Instituto de salubridád $y$ enfermedades tropicales. Biblioteca del Instituto, México, D. F.

Rev. Kuba. Révista Kuba de medicina tropical y parasitologia. $5^{\circ}$ Aventda y calle 78, Habana.

Rov. med. y aliment. Revista de Medicina y alimentación. Soc. Biología. Santiago de Chile, Casilla NQ 38.
Rev. med. brasil. Revista médica brasileira. Rua México, - 164. Río de Janeiro.

Rev. de med. cir. Barranquilla. Revista de medicina y cirugia. Apartado № 202, Barranquilla, Colombia.

Rev. med. Costa Rica. Revista médica de Costa Rica. Apartado 979. San José. Costa Rica

Rev. Med. Cubana. Revista médica cu bana. Apartado 422, Habana.

Rev. med. peruana. Revista médica peruana. Castilla Correo 925, Lima.

Rev. méd. quir. de Oriente. Revista médico-quirúrgica de Oriente. Lacret y Agruilera. Santiago de Cuba. Rev. med. de Rosario. Revista médica de Rosario (República. Argentina). Círculo médico, Calle Italía 663, Ro sario de Santa Fe, Argentina.

Rev. de med. trop. y parasitol., bacteriol. clín. y lab. Revista de medicina tropical y parasitología, bacterio logia, clínica y laboratorio, Apartado 899. Habano.

Rev. med. Hosp. ån. Revista médica del Hospital general. México, D. F. Rev. méd. quir. Revista médico-quirúr. gica. Quito, Ecuador.

Rev. mex. de cir., ginec. y cáncer. Re visia mexicana de cirugía, gineco logía y cáncer. Marslla 11, México D. F.

Rev. mex. de pediat. Revista mexican de pediatria. Bucareli México, I F.

Rev. mex. psiquiat. neurol. y med. ler Revista mexicana de psiquiatris neurología y medicina legat. Gén va; 39, México, D. F.

Rev. mex. de tuberc. Revista mexicar de tuberculosis y enfermedades d apernato respiratorio. Regina $\mathrm{N}$ México, D. F. 
v. neurcl. de Buenos Aires. Revista neurológica de Buenos Aires. Libertad 960 . Buenos Aires.

Rev. neurol e psiquiat. de Sao Paulo. Revisia de neurologia psiquiatria de Sao Paulo. Caixa Postal, 1758. Sao Paulo.

Wy obst y ginec. Caracas. Revista an obstetticia y ginecología. Apartado de Correos 1428, Caracas.

hev. de obst. g ginec, de Sao Paulo. Revista de obstetricia e ginecologia do Sao Paulo. Caixa Postal 168, Sao Ponulo.

Rev. Policlín. Caracas. Revista de la Policlínica Caracas. Apartado 512. Caracas.

Rev. san. de policia. Revista de la sanidad de policia. Casilla № 1683 , Lima.

Rev. Soc. argent. de biol. Revista de la Sociedad argentina de biología. Calle Santo Fe 1171. Buenos Aires.

Rev, urol. México. Revista de urología. Organo oficial de la Sociedad mexi: cana de urologia, Colima 282. Méxt$\infty$, D. F.

PIv. neurol Revista di neurologia. Prof. Mario Gozzano. Clínica neurológica, Polla Saragozza, Bologna.

Focky Nountain M. I. Rocky Mountain Medical Journal. Official Joumal tor Colorado, New Mexico, Uth, Wyoming, Colorado Hospital Association. Rocky Mountaín Medical Conference, 835 Republic Bldg. (1612 Tremont Pl). Denver 2.

S. Clin. North America. Surgical Cli- nies of North America. W. B. Scounders Co, W. Washington Sq. Philadelphia 5.

Sclence. Science. The Scientists Newsweekly. 1515 Masscrchusotts Ave., N. W. Washingtor, 5 D. C.
Scient. Monthly. Scientific Monthly. Secrelary, American Association for the Advancement of Science. Smithsonian Institution Bldg. Washington 25 D. C.

Semana med. La semana médica. Emilio Spinelli, 2240-2248. Calle Córdoba, Buenos Aires.

Soth African J. M. Sc. The South Atrcan Journal of Medical Sciences. Medical School, Hospital St., Johan. nesburg.

St. Barth Hosp. J. St. Barthomew's Hoopital Journal. London, E. C. 1.

Stanford M. Bull Stanford Medical Bulletin. 2560 Bancroft Way, Barkeley.

Surg. Gynec. \& Obst. Surgery, Gyne cology and Obstetries with International Abstract of Surgery. Surgical Publishing Co., 54 E. Erie St., Chicago 11. (British office. 8 Henrietta St. Covent Garden, London, W. C. 2).

Surgery. Surgery. C. V. Mosby Co., 3207 Washington Blvd., St. Louls 3.

Thorax. Thorcax. The Association for the Study of Disecises of the Chest. British Medical Association House, Tavistock Square, London, W. C. I.

Tr. Am. A. Study Coiter. Transactions of the American Association for the Study of Goiter. The Western Journal of Surgery Publishing Co., 305 S. W. Fifth Ave., Portlond 4, Ore.

Tr. Am. Clin. \& Climatol. A. Transactions of the American Cinicai and Climatological Association. Dr. Francis B. Trudeau, 105 Main St., Saranac Lake, N. Y.

Trop. Dis Bull. Tropical Diseases Bulletin. Bureau of Hyglene and Troplcal Diseases, Kappel St., Gower St., London, W. C. 1. 
op. Med. News. Tropical Medicine. Newa. The American Society of Tropical Medicine, National Institut of Heal, Bethesda 14. Md.

iberculólogy. Tuberculology. Official jound of the American Academy of Tuberculosis Physicians. The A ternom Academy of Tuberculosis Ptivsicians, 3800 East Collax Ave. Denver 6.

S. Nav. M. Bull. United States Naval Medical Bulletin. Superintendent of Documents, Government Printign Office, Washington D. C.

iv. Buenos Aires; Misión estud, pat.; reg. argent. Universidad de Buenos Aires Misión de estudios de patologia regional argentina, Juiuy, Universiciad de Buenos Aires. Buenos Aires.

iv. California Publ., Entomol. University of California Publications in Entomology. University of California Press, Berkeley.

iv. California Publ., Physiol. University of California Publications in Phy. siology. University of California Press, Berkeley.

iv. California Publ. Zool. University of California Publications in Zoolo. 9y. University of California Press. Berneley.
Univ. Hosp. Bull., Ann Arbor. University Hospilal Bulletin. University of Michigan, Ann, Arbor.

Vida nuevr. Vida nueva. Manzana de Gómez, 222.23, Habana.

Virchows Arch, f. path. Anat. Wirchows Aechiv fur pathologishe Ana tomie and Physiologie und fur $\mathrm{k} ! \mathrm{l}$ nische Medizsin. Springer Verlag. Neuenheimer Landstrasse 24. Heldelberg.

Wien. Arch. f. inn. Med. Wiener Archiv fur innere Medizin und deren Grenz. gebiete. Urban \& Schwarsenberg. Wien, 71.

Wien klin. Wchnschr. Wiener klinische Wochenschrift. Springer Verlag. Mol. kerbastey 5 , Wien, 1.

Wien med. Wchnschr. Wiener medi. zinische Wochenschrift. Steingasse 25, Wien, 3.

Yale J. Biol. Med. Yale Journal of Biolo. gy and Medicine 333, Cedar St., New Haven.

Ztschr. Kinderpsychat. Zeitschrift fur Kinderpsychatrle. (Journal de psychartrie infantile). Benno Schwabe \& Co., J. Klosterberg 27, basel 10.

Ztschr. 1. Orthop. Zeitschrift fur Ortho padie und ihre Grenzgebiele. Ferdi. nand Enke Verlang, Hasenbergstelge 3 . Stuttgart, W. 